\title{
Alterstice
}

Revue internationale de la recherche interculturelle

International Journal of Intercultural Research

Revista International de la Investigacion Intercultural

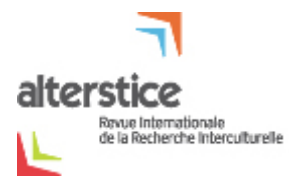

\section{Valladolid : une ligne de fracture interculturelle}

\section{Ygaël Attali}

Volume 5, numéro 1, 2015

URI : https://id.erudit.org/iderudit/1077310ar

DOI : https://doi.org/10.7202/1077310ar

Aller au sommaire du numéro

\section{Éditeur(s)}

Alterstice

ISSN

1923-919X (numérique)

Découvrir la revue

Citer cet article

Attali, Y. (2015). Valladolid : une ligne de fracture interculturelle. Alterstice, 5(1), 83-94. https://doi.org/10.7202/1077310ar

\section{Résumé de l'article}

$\mathrm{La}$ " découverte » $\mathrm{du}$ " Nouveau Monde » à la fin $\mathrm{du} \mathrm{XV}^{\mathrm{e}}$ siècle a été à l'origine de nombreuses interrogations quant au statut religieux, juridique et politique des Amérindiens. La controverse de Valladolid nous offre deux visions opposées concernant l'altérité amérindienne, à partir d’une opposition au sujet de la pratique cannibale. Les regards portés sur cette pratique semblent en effet former le point culminant de la controverse : l'anthropophagie est un archétype à partir duquel tout se joue. Cette réflexion nous permet de développer un modèle dialectique plus global de l'altérité, chaque vision étant source de richesse comme d'affliction. Que penser et que faire face aux comportements radicalement différents des étrangers, surtout lorsque ceux-ci sont condamnés moralement ? C'est aussi à cette question que répondent les deux protagonistes de Valladolid. 
ARTICLE HORS THÈME

\section{Valladolid : une ligne de fracture interculturelle}

Ygaël Attali $^{1}$

\section{Résumé}

La « découverte » du " Nouveau Monde » à la fin du XV siècle a été à l'origine de nombreuses interrogations quant au statut religieux, juridique et politique des Amérindiens. La controverse de Valladolid nous offre deux visions opposées concernant l'altérité amérindienne, à partir d'une opposition au sujet de la pratique cannibale. Les regards portés sur cette pratique semblent en effet former le point culminant de la controverse : I'anthropophagie est un archétype à partir duquel tout se joue. Cette réflexion nous permet de développer un modèle dialectique plus global de l'altérité, chaque vision étant source de richesse comme d'affliction. Que penser et que faire face aux comportements radicalement différents des étrangers, surtout lorsque ceux-ci sont condamnés moralement? C'est aussi à cette question que répondent les deux protagonistes de Valladolid.

\section{Rattachement de l'auteur}

${ }^{1}$ Institut d'études politiques, Paris, France

\section{Correspondance}

ygael.attali@sciencespo.fr

\section{Mots clés}

interculturalité, altérité, identité, barbarie, controverse de Valladolid

\section{Pour citer cet article}

Attali, Y. (2015). Valladolid : une ligne de fracture interculturelle. Alterstice, 5(1), 83-94. 


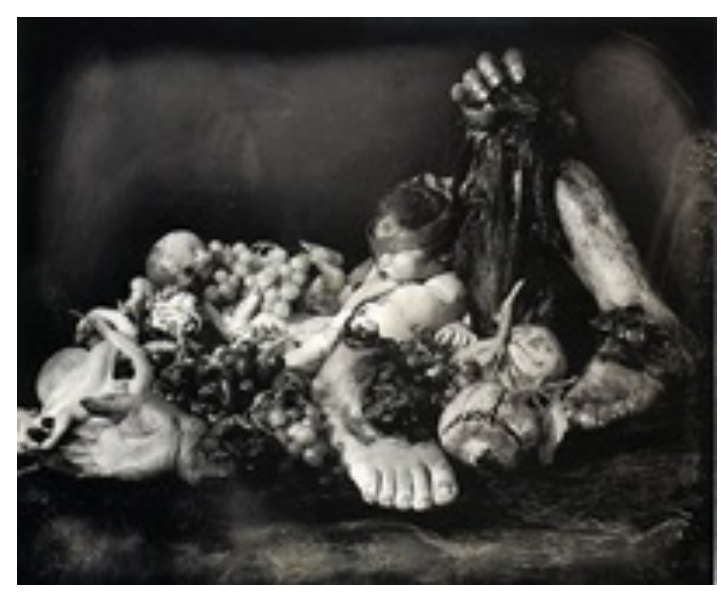

Joel-Peter Witkin, Feast of Fools, 1990

Un plateau de raisins, de mûres et de grenades, photographie faisant référence aux vanités baroques nous rappelant la célèbre locution "memento mori », souviens-toi que tu es mortel. Soudain, l'œil s'attarde sur un pied, puis une main et enfin le corps d'un nourrisson: le choc est violent, impulsif, dénué de toute réflexion philosophique ou artistique. Ce saisissement brutal à la vue de l'œuvre Feast of Fools de Joel-Peter Witkin est sans doute aussi le produit d'une socialisation marquant vivement l'interdiction de toute anthropophagie. Georges Guiles-Escuret, dans Les mangeurs d'autres, civilisation et cannibalisme (2012), souligne avec de multiples exemples historiques comment la notion de civilisation s'est forgée notamment autour du rejet de cette pratique. Cette œuvre nous permet en guise d'introduction de souligner à quel point la tentation ethnocentriste n'est pas uniquement le fait d'un Autre totalement extérieur à nous-mêmes, mais la réaction quasi inéluctable à l'étrangeté. Conséquemment, nous ne souhaitons guère juger du haut de notre humanisme contemporain les diverses positions analysées dans ce travail, mais les étudier à l'aune d'une interrogation jalonnant les études interculturelles: que faire face à "l'étrangeté "? Cette question se pose avec d'autant plus de force lorsque l'étrangeté en question est l'objet d'une condamnation non seulement sociale mais aussi religieuse et morale. Afin d'esquisser une réponse, il est intéressant de tendre l'oreille aux voix spectrales d'un passé semblant lointain, et qui pourtant ouvrent le portail à des interrogations majeures des enjeux sociopolitiques contemporains: la controverse de Valladolid.

\section{Anthropophagie, barbarie et cannibalisme}

Intéressons-nous d'abord au lien ancestral tissé entre cannibalisme et barbarie. Dans le monde hellénique déjà, la prohibition du cannibalisme est comprise comme étant une sortie de notre état animal, problématique qui est exhumée par Alain Schnapp $(1997$, p. 23) :

La justice n'est pas seulement un don de Dieu, elle procède de la nécessité de ne pas pratiquer l'allélophagie qui existe chez les animaux comme dans l'étape sauvage du monde primitif. Les hommes ne pratiquent pas le cannibalisme, ils se détournent de l'habitude qu'avaient les êtres animés de se manger entre eux. Cannibale, l'humanité primitive l'est parce qu'elle ignore les rapports organisés, les relations ordonnées.

En ce sens, note Schnapp, Hésiode serait le premier à donner une définition de ce qui est propre à l'homme à travers ce qui le distingue des animaux : l'interdiction de l'allélophagie ${ }^{1}$. Cependant, le monde grec sera lui aussi marqué par des dissonances, et parfois même des aboiements violents interrompant, avec une insolence et une obscénité revendiquées, les nomes normés grecs. Ainsi, désireux de briser le modèle de la cité, les Cyniques revendiqueront avec fougue le cannibalisme, et l'oiseau, qui était dans l'imaginaire grec le symbole des excès du monde sauvage (Hésiode opposera la loi humaine aux « oiseaux ailés » qui se dévorent), sera désigné comme un

\footnotetext{
${ }^{1}$ « Pour toi, Persès, mets-toi ces avis à l'esprit; écoute la justice, oublie la violence à jamais. Telle est la loi que le cronide a prescrite aux hommes: que les poissons, les fauves, les oiseaux ailés se dévorent puisqu'il n'est point parmi eux de justice. Mais aux hommes Zeus a fait le don de la justice qui est de beaucoup le premier des biens. »
} 
modèle, en particulier par le coq (Auger, Rosellini, Sal̈d et Vidal-Naquet, 1979, p. 84). L'animalité de l'homme n'est, dans ce cas, guère combattue, plutôt fièrement revendiquée. Notons conséquemment que ce n'est pas l'affirmation d'une pluralité de modèles de civilisation qui est défendue, mais bien une attaque symbolique forte de l'idée même de civilisation ${ }^{2}$. Cette dissonance demeure intéressante à garder en mémore afin de peindre le monde grec dans toute sa polychromie et de souligner que les interrogations centrales posées par la controverse de Valladolid transcendent les frontières et les siècles.

Si le rejet de l'anthropophagie est une constante dans le monde chrétien occidental, c'est avec la " découverte » de l'Amérique que cette thématique prendra une teinte particulière. Rappelons que le terme même de cannibalisme sera forgé par Christophe Colomb à partir du mot caniba employé par les Taïnos qu'il a rencontrés lors de son premier séjour sur Hispaniola. Le cannibalisme rejoindra d'autres mythes - tels ceux des cyclopes, des cynocéphales ou des sirènes - supposés exister dans ces contrées sauvages. Si le regard porté sur l'anthropophagie a longtemps relevé d'une mer agitée séparant les rives sombres de la barbarie et celles lumineuses de la civilisation, certains auteurs ont, à différentes époques, remis en question la pertinence de cette césure. La controverse de Valladolid est une illustration intéressante des différentes conceptions et aprioris susceptibles de graviter autour de cette pratique sociale et, à travers elle, de la vision portée sur l'étrangeté. Un rappel du contexte historique de la controverse va nous permettre de relever les principaux arguments de chacun, en soulignant la place particulière du cannibalisme et du sacrifice humain, éléments paroxystiques de la célèbre dispute. Nous élaborerons ensuite une réflexion critique de la pensée des deux opposants à l'aune de la question de l'étrangeté, posée constamment dans un contexte interculturel.

\section{Contexte de la controverse de Vallodolid}

Une mise en contexte historique semble nécessaire afin d'appréhender la célèbre controverse de Valladolid opposant deux éminents penseurs religieux du XVI siècle, Bartolomé de Las Casas (1474-1566) et Juan Ginés de Sepúlveda (v. 1490-1573). Contextualisation précieuse car, orpheline des trames l'ayant structurée, cette controverse ne peut être comprise ni dans les arguments échangés ni dans les conséquences théoriques de cette confrontation de pensées profondément antinomiques. Liée à un contexte économique, social, religieux et politique particulier, elle nécessite une clarification historique, mais, faute de place et d'expertise, nous n'en exposerons pas tous les tenants et aboutissants.

En 1492, les Espagnols débarquent pour la première fois sur un "nouveau continent ", que Christophe Colomb croyait être les Indes orientales, région tant convoitée pour ses richesses et épices précieuses. La Couronne d’Espagne prendra possession de ces contrées vantées par Colomb, terres qu'elle nommera Nouvelle Espagne puis baptisera « Amérique » en 1507 en hommage au navigateur florentin Amerigo Vespucci. La population autochtone, que l'on nomme à l'époque les "Indiens ", va voir ses conditions de vie bouleversées par des pillages, commerces d'esclaves, massacres, humiliations, profanations et mises à sac de pans entiers de leurs cultures. Rappelons en outre que ces cultures locales multiples ont été vues dans un premier temps comme étant homogènes, signe initial du mépris et de l'ethnocentrisme escortant les conquêtes. De nombreuses exactions ont été décrites avec précision par Las Casas (voir Las Casas, 1979) et d'autres missionnaires présents sur le terrain à cette époque, tel Antonio de Montesinos, homme ayant beaucoup influencé le premier dans la dénonciation vibrante de ces massacres ${ }^{3}$. Mue notamment par des intérêts économiques liés à la plantation de champs agricoles et une recherche de ressources naturelles (en particulier or et argent), une entreprise coloniale et esclavagiste va se développer au cours du XVI siècle, et la conquête du nouveau continent se fera au prix de millions de morts (Dickinson et Mahn-Lot, 1991).

\footnotetext{
${ }^{2}$ Sur cette question, les analyses de Marcel Détienne sont particulièrement éclairantes (voir Détienne, 1996).

${ }^{3}$ Sermon de Montesinos: "Je suis la voix de Celui qui crie dans le désert de cette île et c'est pour cela qu'il faut que vous m'écoutiez avec attention Cette voix est la plus neuve que vous ayez jamais entendue, la plus âpre et la plus dure. Cette voix vous dit que vous êtes tous en état de péché mortel; dans le péché vous vivez et vous mourrez à cause de la cruauté et la tyrannie dont vous accablez cette race innocente. Dites-moi, quel droit et quelle justice vous autorisent à maintenir les Indiens dans une aussi affreuse servitude? Au nom de quelle autorité avez-vous engagé de telles détestables guerres contre ces peuples qui vivaient dans leurs terres d'une manière douce et pacifique, où un nombre considérable d'entre eux ont été détruits par vous et sont morts d'une manière encore jamais vue tant elle est atroce ? [...] Ne sont-ils pas des hommes ? Ne sont-ils pas des êtres humains ? Ne devez-vous pas les aimer comme vous-mêmes ? " (Las Casas, 2002, p. 26-27).
} 
Bien que les chiffres fassent l'objet d'un vif débat historique - principalement lié à la controverse concernant la qualification de génocide (Dorel, 2006) -, un consensus existe sur le fait que plus de la moitié de la population autochtone a péri au cours des trois siècles suivant la découverte du continent (Russel, 1990). Rappelons cependant que la majorité des décès ont été causés par des maladies, une dépopulation intentionnelle aussi rapide - même si les massacres ont été multiples et importants - étant techniquement impossible. C'est ainsi que la variole, la tuberculose, le typhus, mais également la grippe, la rougeole et la diphtérie sont, dès octobre 1492 , les agents principaux de la disparition de millions d'individus. De nombreuses tribus seront ainsi convaincues par ces faits dramatiques d'une trahison divine, ce qui facilitera grandement le travail des « tout puissants » missionnaires (Dorel, 2006).

Rappelons, enfin, l'importance des Lois nouvelles dans le contexte de la controverse de Valladolid: Marianne Mahn-Lot soulignera l'influence cruciale de Las Casas dans l'élaboration de celles-ci (Mahn-Lot, 1990). Charles Quint, ayant autorisé l'esclavage des Indiens en 1517, en interdit la pratique en 1526 afin " que les Indiens soient libres et non soumis à la servitude » (cité par Lavallé, 2007, p. 183). Le pape Paul III confirme ce jugement par deux bulles successives en 1537, condamnant l'esclavage des Indiens et confirmant leur droit à la liberté et à la propriété. En 1549, l’Audience de Pérou fut informée que désormais la pénétration en territoire indien devrait se faire de façon pacifique. Cette orientation demeurera la position officielle de Madrid et de Rome. Notons cependant que les textes officiels ne seront pas ou peu respectés par les potentats locaux, et les couronnes se rendront coupables d'un laisser-faire parfois réprobateur, souvent complaisant. Rappeler cela permet de souligner que les idées défendues par Las Casas, bien avant la célèbre controverse que nous allons étudier, constituaient en partie la doctrine officielle de l'Église et de l'État et qu'elles commençaient à s'inscrire dans les faits législatifs et politiques de l'époque (Lavallé, 2007).

La contextualisation historique et politique permet d'entrer dans le vif de la controverse célèbre, Lewis Hanke, l'un des spécialistes de cette période, affirmant que celle-ci est « un des événements les plus curieux de l'histoire du monde occidental dans la mesure où pour la première fois, et sans doute la dernière, un empire organisa officiellement une enquête sur la justice des méthodes employées pour étendre ses domaines » (Hanke, 1957, p. 178). Elle est à notre avis exceptionnelle aussi par la qualité du débat ayant eu lieu, qui a permis d'établir deux positions profondément opposées à ce sujet et s'est fondé sur des prémisses à partir desquelles nous pouvons comprendre la conceptualisation de la barbarie à travers le fait anthropophagique ainsi que - et surtout - de nombreux désaccords à propos du rapport de l'être humain à l'altérité.

\section{Les protagonistes}

La controverse eut lieu à Valladolid à partir du mois de juillet 1550 et dura plus d'un an, tout en étant interrompue à plusieurs reprises. Quatorze personnalités de premier plan y ont été réunies : des membres du Conseil des Indes et de celui de Castille, des hauts fonctionnaires ainsi que des théologiens franciscains et dominicains reconnus ${ }^{4}$. La junte fut chargée d'étudier «la manière dont notre sainte foi catholique doit être prêchée et publiée au Nouveau Monde; à quelles conditions ces peuples demeureront sujets de Sa Majesté sans que sa conscience royale en souffre, et conformément à la bulle de Sa Sainteté Alexandre VI » (cité dans Bénat-Tachot et Lavallé, 2005, p. 224). Quels sont l'ordre et la forme convenable de la conquête? La question de la forme légitime de l'empire chrétien en terre américaine est donc posée, et avec elle s'ouvre la brèche de la légitimité même des conquêtes espagnoles. Une interrogation sur ses modalités a donné naissance à une controverse sur le modèle lui-même, et la réflexion sur l'étendue et les fondements des moyens a éclaboussé la pureté affirmée des fins.

Pour l'exposé des motifs, Sepúlveda et Las Casas furent conviés à présenter leurs idées séparément, chacun étant absent de l'intervention de son contradicteur, contrairement à ce que l'on imagine souvent, notamment dans les créations littéraires ultérieures ${ }^{5}$. D'ailleurs, ces dernières ont aussi popularisé l'idée selon laquelle les Espagnols

\footnotetext{
${ }^{4}$ Citons les dominicains Cano, Carranza, Soto et le franciscain Arévalo.

${ }^{5}$ L'image d'une controverse où l'échange d'arguments vifs se fait face à face est souvent empruntée aux fictions l'ayant dépeinte, par exemple la célèbre œuvre théâtrale de Jean-Claude Carrière, La Controverse de Valladolid (1992).
} 
débattaient de l'existence de l'âme des Indiens, alors que la volonté même de les évangéliser prouve leur pleine appartenance à l'humanité dans les consciences dominantes de l'époque.

Né à Séville en 1484 dans une famille probablement marrane, Las Casas est un " Espagnol moyen " (Capdevila, 2007) sans formation universitaire, dont les connaissances juridiques et théologiques ont été tardivement acquises de manière autodidacte. Sepúlveda, né en 1490 dans une famille noble près de Cordoue, a étudié la philosophie à I'université d'Alcala puis la théologie à Sigüenza. Après avoir servi Clément VII, il devient chapelain de Charles Quint puis précepteur du prince Philippe. Alors que Sepúlveda intervient dans les controverses majeures de son temps en défendant Catherine d'Aragon répudiée par Henri VIII, en critiquant Luther et Érasme ou en rédigeant la chronique du règne de Philippe II, les écrits de Las Casas portent pour leur part quasi exclusivement sur l'Amérique.

Sepúlvede s'inspire fortement des arguments exposés dans l'un des ses ouvrages clé, Democrates alter, sive De justis belli causis contra indio (Des justes causes de guerre contre les Indiens). Étant l'un des plus grands savants hellénistes de son époque, il s'appuie beaucoup sur la Politique d'Aristote (qu'il traduit en latin) et son concept de « servitude naturelle ». Las Casas reprend pour sa part l'argumentation développée dans les Principia Quadum.

\section{La vision de Sepúlveda : en guerre contre l’anthropophagie}

Un terme employé par Sepúlveda pour qualifier l'Indien et expliquer l'attitude à adopter envers lui est extrêmement significatif et révélateur: il s'agit de hommunculus, diminutif de homo. Autrement dit, l'Indien n'était, comme le disait la version espagnole du texte, qu'un hombrecillo, un " petit homme » ou " sous-homme ». L'humanité des Indiens proclamée par le pape était donc ainsi reconnue, mais en même temps tempérée pour permettre au système colonial de s'exercer sans entraves. Sepúlveda, suivant l'enseignement de son maître penseur ${ }^{6}$, croit que la hiérarchie est l'état naturel de la société humaine. Le corps doit être soumis à l'âme, la matière à la forme, les enfants aux parents, la femme à l'homme et les esclaves aux maîtres : certains naissent pour commander, d'autres pour obéir. Un autre texte auquel il se réfère couramment est un traité, De regimine principum, attribué à l'époque à saint Thomas d'Aquin mais dû en réalité à Ptolémée de Lucques (Todorov, 1982), qui ajoute à l'affirmation de l'inégalité une explication déjà ancienne mais encore promise à un grand avenir : il faut en chercher la raison dans l'influence du climat et dans celle des astres. À partir de cette vision inégalitaire de l'être humain, l'argumentaire de Sepúlveda se divise en quatre propositions légitimant une guerre contre les Indiens : ils ont une nature soumise et intellectuellement inférieure, ils sont idolâtres, pratiquent le cannibalisme et sacrifient des êtres humains ${ }^{7}$, ces trois derniers faits étant une preuve et une conséquence de la première proposition affirmant leur infériorité naturelle, leur statut d'hommunculus : dénués de raison naturelle, leurs comportements sont forcément barbares, animaliers, sauvages ${ }^{8}$. Or le paroxysme de la barbarie n'est-il pas justement le sacrifice humain et le cannibalisme? Étant étrangers à l’Église, les Indiens échappent à sa juridiction, mais la loi naturelle

\footnotetext{
${ }^{6}$ "La seconde société se forme entre deux individus que la nature a faits, I'un pour commander, l'autre pour obéir. Ils se réunissent pour leur mutuelle conservation. L'homme qui a la force de l'entendement et de la prudence, a reçu de la nature le commandement et l'empire. Celui qui n'a que la force du corps pour exécuter, est par l'ordre de la nature obéissant et esclave. Le maître et l'esclave trouvent donc dans leur réunion un commun avantage. " (Aristote, Politique, Livre I, traduction française de J.-F. Champagne, revue et corrigée par M. Hoefer).

${ }^{7}$ Extrait du résumé officiel de la controverse, le Sumario de Soto (Las Casas, 1991, p. 182) : « Le docteur Sepulveda appuie brièvement son affirmation sur quatre raisons :

1. la gravité des délits des Indiens, principalement leur idolâtrie et leurs péchés contre nature,

2. la grossièreté de leur intelligence qui en fait une nation servile et barbare, destinée à être placée sous l'obédience d'hommes plus évolués comme le sont les Espagnols,

3. les besoins mêmes de la foi, car leur sujétion rendra plus facile et expédiente la prédication qui leur sera faite,

4. les maux qu'ils s'infligent les uns aux autres, tuant des hommes pour les offrir en sacrifice ou pour les manger. »

${ }^{8}$ Une présentation complète de l'argumentation des deux protagonistes est faite dans Capdevila (2007).
} 
étant universelle, ils retombent en réalité sous ses injonctions par ce biais. Or le cannibalisme incarne pour Sepúlveda ce « qui fait le plus de violence à la nature »" ${ }^{9}$.

La sodomie, les meurtres rituels, mais aussi et surtout l'anthropophagie sont, dans les sociétés amérindiennes - vus sous l'angle homogénéisateur de Sepúlveda - des caractéristiques qui les définissent. Le meurtre, par exemple, existe bel et bien en terre chrétienne, mais la différence fondamentale forgée au cours de son œuvre se situe sur le terrain de l'institutionnalisation de comportements "contre-nature » (cité dans Capdevila, 2007, p. 186). L'homicide, l'adultère et le vol sont les fruits pourris de toutes les nations, mais en contraste avec la sienne, celle des Amérindiens sanctifie le crime en lui donnant un sens socioreligieux. Le fruit du crime est ici consacré et célébré par tous. Cet argument lui permet de contrecarrer Las Casas et d'autres auteurs ayant souligné le caractère ritualisé à l'extrême de l'anthropophagie, voulant offrir pour leur part une facette lumineuse dans le puits obscur du cannibalisme. Sepúlveda prend donc le contre-pied de cet argument en montrant que c'est justement l'institutionnalisation qui fonde la barbarie paroxystique de l'acte cannibale. La distinction entre culpabilité individuelle et culpabilité de la nation permet au procureur Sepúlveda de réclamer la condamnation sans appel de cette dernière : le crime n'étant pas puni par les caciques locaux, ce devoir incombe aux "nations civilisées ". D’ailleurs, le devoir ne se limite pas simplement au châtiment, l'épreuve est plus large: l'ingérence pour le sauvetage de vies innocentes. En estimant le nombre de sacrifices humains à 20000 par an, Sepúlveda souligne que la guerre de conquête épargnera des vies humaines. Si le théologien exagère ce chiffre ${ }^{10}$, Las Casas n'hésitera pas pour sa part à le minimiser en répliquant " ni cent ni cinquante par an " (cité dans Capdevila, 2007, p. 266), faisant preuve d'aveuglement partisan.

\section{La vision de Las Casas : promouvoir une conversion pacifique}

Las Casas, comme son adversaire, a comme source de pensée principale les écrits religieux et antiques, mais il en tire une vision diamétralement opposée. L’Évangile, soutient-t-il, doit être prêché de manière pacifique, grâce à la conviction et non dans la contrainte et la violence. Levant la justification d'une coercition nécessaire à l'évangélisation, Las Casas va plus loin dans son cheminement intellectuel puisqu'il soutient que les peuples amérindiens ne sont ni barbares ni primitifs et ne correspondent donc en rien aux définitions des serfs a natura dont parlait Aristote : leur culture et leurs mœurs diffèrent, mais ils sont essentiellement similaires aux chrétiens venant prêcher leur foi. C'est à partir de cette notion égalisatrice d'identité humaine que Las Casas peut bâtir une identification à l'Autre : le nivellement permet, dans un premier temps au moins, l'identité. Cette dernière est une toute autre approche à l'altérité puisqu'elle se focalisera sur les points de jonction identitaires. Une explication voire une justification de nombreux aspects de la culture étrangère étant analysées avec l'œil bienveillant du penseur ayant trouvé un frère humain, l'analogie sera donc une figure rhétorique maîtresse dans l'échiquier du prêtre dominicain. Cela lui permet de défendre différents us et coutumes comme la nudité (résultant du climat), l'idolâtrie (résultant de l'ignorance à combler) ou encore les guerres internes (existant aussi au sein des royaumes chrétiens). Le point culminant du désaccord résultant d'une antinomie des principes sera atteint avec le sacrifice humain et l'anthropophagie.

Si la conception hiérarchique de Sepúlveda pouvait être placée sous le patronage d’Aristote, la conception égalitariste de Las Casas mérite d'être présentée comme issue principalement de la perspective thomiste du Christ. Il dit lui-même dans son discours de Valladolid : "Adieu, Aristote! Le Christ, qui est la vérité éternelle, nous a laissé ce commandement: "tu aimeras ton prochain comme toi-même" " (cité dans Todorov, 1982, p. 204). À l'égalitarisme s'ajoute l'affirmation d'une conversion qui doit se faire sous la persuasion et non la contrainte. Citant à ce titre Thomas d'Aquin, il écrit « croire est un acte de la volonté libre, [...] donner la foi de force, c'est forcer la justice " (Las Casas, 1991, p. 36). Injuste, une conversion contrainte est également inefficace à ses yeux, puisque la religion, comme la science, doit être embrassée par la raison humaine : un esprit véritablement libre adhérera

\footnotetext{
${ }^{9}$ " La deuxième cause [légitimant la guerre] est la suppression du crime monstrueux de dévorer la chair humaine qui fait le plus de violence à la nature et d'empêcher que les démons ne soient honorés à la place de Dieu, ce qui provoque le plus sa colère, avec le rite monstrueux de sacrifier des victimes humaines " (Sepúlveda, cité dans Capdevila, 2007, p. 168). Ce qui outrage la nature elle-même, et l'élément central à partir duquel la barbarie indienne est affirmée, est bien l'anthropophagie.

${ }^{10}$ Concernant la controverse, encore actuelle, sur les chiffres des sacrifices humains, voir l'ouvrage extrêmement complet de Graulich (2005).
}

Alterstice - Revue Internationale de la Recherche Interculturelle, vol. 5, $n^{\circ} 1$ 
corps et âme, une personne asservie le fera avec regret et amertume et le monde que cela instaurerait sera un univers dominé uniquement par la force, non par les lumières de la « vérité divine » $(p .37)^{11}$.

\section{Des visions divergentes de l'étrangeté}

La double figure de la pureté innocente et de la ressemblance à soi ${ }^{12}$ poussera Las Casas à défendre les Amérindiens sur le terrain le plus épineux, celui du cannibalisme. C'est un point rocailleux et pourtant culminant du mont de Valladolid, puisqu'il oppose diamétralement les deux protagonistes et permet de comprendre la divergence : chacun observe une autre face du col.

Bartolomé de Las Casas, prêtre dominicain et missionnaire, va dans un premier temps expliquer l'idolâtrie : comme les idolâtres " pensent et estiment que ces idoles sont le vrai Dieu » (cité dans Capdevila, 2007, p. 188), l'objet véritable de leur amour et de leur pensée n'est pas l'idole, mais Dieu. Or ce dernier doit être honoré avec l'offrande la plus précieuse aux yeux des dévots, la valeur variant entre les sociétés et chacune ayant la légitimité de juger du présent à offrir. Tous les membres d'un État ont ainsi l'obligation de pratiquer cette forme d'amour de Dieu et de le défendre, car le droit naturel leur impose d'obéir aux autorités légitimes. L'offrande sacrificielle, puisque c'est évidemment de cela qu'il s'agit, n'est pas seulement faite pour Dieu mais pour le " bien de toute la république ». C'est pourquoi le sacrifice humain " n'est pas aussi contraire à la raison naturelle que s'il s'agissait d'une chose abominable immédiatement contraire à un précepte de la nature " (cité par Capdevila, 2007, p. 188). Ainsi, le sacrifice humain est une façon raisonnable de révérer Dieu. La question de l'institutionnalisation de cette pratique est ainsi totalement renversée : si elle est raisonnable, sa perpétuation officialisée n'a rien de condamnable en soi dans l'infra-logique de la culture en place - et ainsi cet argument est invalidé, il ne prouve plus la barbarie des Indiens. D'ailleurs, si le sacrifice humain contredisait la loi naturelle, Dieu n'aurait pu formuler l'ordre de sacrifier Isaac. Le sacrifice humain est bien entendu condamnable, mais en raison d'une foi chrétienne dont ne disposent pas les Indiens, non en raison d'une quelconque loi naturelle. D'ailleurs, on trouve des témoignages de sacrifices humains dans toute l'Antiquité. Si ici nous ne distinguons pas clairement le sacrifice humain et l'anthropophagie, c'est tout simplement parce qu'ils sont souvent liés dans les argumentations des deux opposants.

Le point culminant de la barbarie est-il l'emploi d'un être humain comme moyen pour une fin religieuse ou alimentaire ou, comme souvent, une juxtaposition complexe des deux? Citons Nestor Capdvilla, qui a parfaitement synthétisé l'enjeu de la dispute :

Sepúlveda soutient que l'idolâtrie et le sacrifice humain contredisent la loi naturelle. Mais du point de vue de Las Casas, c'est exiger de la raison une perfection qu'elle ne peut tenir que de la foi. L'erreur de Sepúlveda est donc de projeter dans la loi naturelle une perfection surnaturelle en méconnaissant la différence entre la foi et la raison, la nature et la grâce. (Capdvilla, 2007, p. 189).

\footnotetext{
11 « si au lieu de la suavité de la voix, d'une attitude pleine de modestie, de l'expression de la douceur, du choix paisible des mots, de tout ce qui, en un mot, peut faire naître plaisir et affections; si, donc, au lieu de tous ces attraits, on ne rencontre que clameurs continuelles et haineuses, visages terrifiants, regards farouches et démoniaques, fureurs colériques d'hommes frénétiques semblables à des animaux sans raison et plein de fiel, paroles d'affronts et d'injures, bruit sinistre d'armes s'entrechoquant, semant partout les cadavres [...] comment, dans ces conditions sera-t-il possible d'amener les âmes à écouter les vérités de la foi et de la religion ? Où sont les délicatesses, les attraits qui peuvent les disposer à l'attention et à la docilité ? [...] Bien plus, ceux qui auront eu à souffrir de telles choses adopteront, pour toujours et à juste titre, une attitude inexorable, malveillante, indocile, refusant d'écouter quoi que ce soit qui ait rapport au nom de chrétien, et deviendront les ennemis de ce nom. " (cité dans Las Casas, 1991).

12 Une ressemblance concernant notamment la capacité intellectuelle et l'usage de la raison: "Les Indiens ont d'aussi bons entendements, des esprits aussi perspicaces, aussi capables et dociles pour n'importe quelle science morale et doctrine spéculative; pour la majeure partie, ils sont aussi ordonnés, aussi pourvus et raisonnables dans leur police - car ils ont beaucoup de lois très justes - et ils ont tiré et tirent tous les jours autant de profit des choses de la foi et de la religion chrétienne, relativement aux bonnes coutumes et à la correction des vices, partout où ils ont été instruits par les religieux et des personnes de bonne vie [...] et je ne parle pas de leurs admirables progrès dans les arts mécaniques et les arts libéraux, comme la lecture et l'écriture, la musique vocale, la pratique de tous les instruments de musique, la grammaire, la logique, et dans tout le reste de ce qui leur a été enseigné et qu'ils ont écouté ". Ici encore, c'est à l'aune des arts et de la culture chrétienne que les indiens sont jugés (positivement) quant à leurs capacités naturelles.
} 
La rationalisation du sacrifice humain va faire le pont avec l'anthropophagie qui lui est liée : bien que « horrible et abominable", pourquoi serait-elle plus blâmable que le sacrifice puisqu'elle est faite "par religion et non pour autre chose " (Capdvilla, 2007, p. 190)? Consommée " comme une chose sacrée ", la chair des sacrifiés avait selon eux le pouvoir de les sanctifier, raison pour laquelle le banquet anthropophage est qualifié de "réjouissant " (Capdvilla, 2007, p. 193).

Cette vision synthétique de la " dispute " - au sens médiéval et universitaire du terme - permet de comprendre qu'aux racines du dense débat ayant opposé deux pensées concurrentes, la vision de l'Autre (Levinas, 2000) diffère : pour Sepúlveda, l'infériorité naturelle infère aux Indiens des qualités n'existant pas dans la vision de Las Casas, du fait même de leur égalité par essence pour ce dernier. L'interprétation de leurs faits et coutumes diffère donc en fonction des prémisses sur lesquels la vision de l'Homme est bâtie. Leurs positions réciproques sur le cannibalisme et le sacrifice humain sont emblématiques de cette différence fondamentale dans les axiomes sur lesquels chacun se fonde: Sepúlveda, sur un regard hiérarchisant qui interprète ces pratiques sous l'angle de la barbarie, Las Casas, sur une perspective arasante qui identifie l'Autre à soi et aplanit totalement l'étrangeté et ses particularités.

Dans les mois suivant la controverse, les deux protagonistes et leurs soutiens se sont proclamés vainqueurs. En réalité, aucun texte, aucune déclaration ne vint jamais dire officiellement quelle était en la matière la position de la Couronne. II semblerait que les personnalités présentes lors de la dispute soient demeurées divisées à la fin de celle-ci. Bernard Lavallé (2007) rappelle cependant que

[les juntas organisés par la Couronne] sur des sujets sensibles et complexes n'avaient pour objet que de susciter des débats de fond entre spécialistes sur telle ou telle question, de faire en quelque sorte le point, et de l'informer. Elle ne se sentait cependant en aucune façon liée par leurs conclusions, et nombreux furent les cas où elle se décida en définitive d'une façon contraire à celle qu'on lui avait suggérée.

Marianne Mahn-Lot considère pour sa part que Sepúlveda fut vaincu dans les faits à la suite du débat: son Democrates secundus n'obtint jamais licence d'être imprimé en Espagne et les guerres de conquête furent partiellement suspendues et le mot même de "conquête » plus tard aboli ${ }^{13}$, suivant ainsi les conseils de Las Casas qui traitait ce terme de «mahométan et inique » (Las Casas, 1991, p. 59). Si cet abandon du terme est motivé par une conception non militaire de l'appropriation des terres et semble signer la victoire de Las Casas, l'euphémisation linguistique, bien que dénotant une certaine évolution des consciences, ne signifie aucunement l'abandon de la logique du Requerimiento ${ }^{14}$ et la poursuite en conséquence de la colonisation. Nestor Capdevila en conclut donc plutôt que Las Casas et Sepúlveda ont en réalité tous les deux perdu (Capdevila, 2007, p. 141).

\section{Égalité, identité et altérité}

S'il est incontestable que le préjugé de supériorité incarné par Sepúlveda est un obstacle dans la voie de la connaissance et de l'établissement de liens avec autrui, il faut aussi admettre avec Tzvetan Todorov que le préjugé d'égalité et d'identité est tout aussi préjudiciable, car il « consiste à identifier purement et simplement l'Autre avec son propre moi » (Todorov, 1982, p. 171). Le postulat d'égalité entraîne chez Las Casas l'affirmation d'identité : si cela renforce considérablement le propos rhétorique de l'auteur, cela fragilise du même coup sa capacité à voir les Amérindiens dans leurs particularités. L'identité, cette seconde grande figure de l'altérité, conduit ainsi à une méconnaissance de l'Autre, puisque la caractérisation d'autrui est indépendante de ses qualités propres: on ne voit pas l'Autre tel qu'il est, mais on se projette en miroir dans celui-ci, et on réduit de ce fait ses particularités à des détails à mutiler afin de considérer l'Autre dans sa similitude à nos propres qualités. Dans ce cadre, la justification du cannibalisme ne se fait pas par des arguments de relativisme culturel ou de respect de cultures différentes, mais sous l'angle exclusif de la ressemblance à soi et de la justification dans une logique chrétienne: c'est parce que l'acte de dévotion est immense et que l'offrande a une valeur importante qu'ils sont légitimes, puisque la divinité est l'entité qui prime. On abolit les différences entre les idoles et le Dieu chrétien, entre la prière

\footnotetext{
${ }^{13}$ Le terme " conquista » est absent dans les Leyes Nuevas de 1542 et les instructions de 1556, et il disparaîtra du vocabulaire officiel dans le chapitre 29 des ordonnances de 1573 (Capdevila, 2007, p. 139).

${ }^{14}$ Texte juridique rédigé en 1512 par Juan Lopez de Palacios Rubios légitimant les conquêtes.
} 
et l'eucharistie d'une part et le cannibalisme de l'Autre : les disparités sont passées sous silence pour mettre en lumière les correspondances et analogies.

L'Amérindien n'est guère respecté ici pour ce qu'il est dans sa différence, mais grâce à ses ressemblances à « nos » propres coutumes. À ce titre, l'un des passages de la dispute est extrêmement révélateur : il existerait selon Las Casas trois catégories de barbares :

La première [catégorie], dans un sens large, s'applique à tout peuple qui a des opinions et des coutumes étranges, bien qu'il soit néanmoins policé et qu'il sache se gouverner. La seconde catégorie s'applique aux peuples qui n’ont pas de langages écrits se traduisant par des caractères ou par des lettres. [...] La troisième catégorie de barbares s'applique aux peuples que leurs coutumes perverses, la grossièreté de leur esprit et leurs inclinations brutales apparentent aux animaux sauvages qui vivent dans les plaines, sans villes, sans maison, sans ordre policé, sans loi, sans rites ni traités [...], se livrant au vol et à la violence comme firent au début les Goths et les Alains et, aujourd'hui, les Arabes d'Asie à ce qu'on dit, et ceux d'Afrique que nous appelons Alarabes. À cette sorte de gens, on pourrait appliquer ce que dit Aristote : il serait licite de leur faire la guerre comme on chasse les bêtes féroces, pour nous défendre quand ils nous attaquent, et en nous efforçant de les amener à une façon de vivre plus humaine ». (cité dans Las Casas, 1991, p. 185)

Les Indiens eux, ont

" de gros bourgs, des maisons, des lois, des seigneurs, pratiquent des arts, punissent les pêchés contre nature de peine de mort. Ils ont donc un degré suffisant de vie policée, et l'on n'est pas en droit de leur faire la guerre. " (cité dans Las Casas, 1991, p. 185).

Les Indiens ne sont donc pas des barbares parce que l'on doit comprendre de l'intérieur leur culture étrangère et leurs particularités, mais parce qu'ils correspondent à nos critères de civilité. Rappelons que le premier grand traité de Las Casas consacré à la cause des Indiens s'intitule De l'unique manière d'attirer tous les peuples à la vraie religion, ce titre condensant en lui l'ambivalence de la position de l'auteu : les peuples sont vus dans leur unité homogénéisatrice, et la religion chrétienne pourrait et devrait les envelopper identiquement. Selon Tsvetan Todorov (1982, p. 182) :

Personne n'a su comme lui, avec la même abnégation, consacrer une immense énergie et un demi-siècle de sa vie à améliorer le sort des autres. Mais cela n'enlève rien à la grandeur du personnage, bien au contraire, de reconnaître que l'idéologie assumée par Las Casas et d'autres défenseurs des Indiens est bien une idéologie colonialiste. C'est justement parce qu'on ne peut s'empêcher d'admirer l'homme qu'il importe de juger lucidement sa politique.

Las Casas et d'autres défenseurs des Indiens ne sont pas hostiles à l'expansion espagnole, mais ils préfèrent l'une de ses formes à l'autre. La vision " arasante " comme la vision prônant l'inégalité ont une face sombre ayant mené à des dérives. La controverse de Valladolid a permis à l'un des plus grands humanistes d'exprimer une défense touchante et essentielle des peuples amérindiens, mais une étude approfondie de sa vision permet d'en souligner les zones d'ombre et ainsi d'illustrer deux pensées fondamentalement opposées ouvrant la voie à une appréhension dialectique de la question de l'altérité. Notons que cette relecture de la controverse de Valladolid n'est en aucun cas une critique anachronique des positions de l'un ou de l'autre des adversaires, et donc que nous n'emploierons pas le terme d' "idéologie colonialiste » à ce sujet. Ce concept, rattaché par Todorov à la pensée de Las Casas, est encore moins pertinent lorsque l'on connaît l'extrait suivant d'une lettre de ce dernier à Charles Quint :

Pour être de bons chrétiens, ils devraient tous comprendre que même si Votre Majesté pouvait perdre entièrement ladite seigneurie royale et que les Indiens ne devenaient jamais chrétiens, il ne serait pas dommageable que Votre Majesté cessât d'être leur seigneur et que les Indiens ne devinssent jamais chrétiens [...] Vouloir tuer les infidèles sous prétexte de les 
sauver, ou en tuer quelques-uns pour sauver les autres, sont de grands maux et des péchés inexpiables dignes de la damnation éternelle. Dieu ne veut pas un tel gain avec une telle perte ». (cité dans Capdevila, p. $197^{15}$ )

La défense des vies indiennes supplante ici la nécessité de l'empire et même celle de la conversion. Si logique coloniale il y a, elle est dénuée de toute violence, même minime.

\section{Enjeux de l'interculturalité}

Cette controverse nous permet de nous saisir d'enjeux plus généraux à propos de l'interculturalité. Une vision de l'Autre comme étant totalement et diamétralement différent (vision incarnée ici par Sepúlveda) ne permet évidemment pas la mise en place de fondements communs permettant la compréhension de certaines coutumes comme le cannibalisme, mais une vision de l'Autre comme étant identique à soi (vision de Las Casas) tend à mutiler ses différences et l'originalité de son identité et ne permet alors pas non plus une véritable reconnaissance de la partie adverse. Si Sepúlveda pèche par excès de différenciation et par un rejet total et hiérarchisant des particularités étrangères, Las Casas semble être, avec son point de vue arasant, dans un excès inverse. Trois raisons, perceptibles dans la controverse analysée ici, appuient ce point: ethnocentrisme, assimilationnisme et aveuglement parcellaire.

Si l'ethnocentrisme de Sepúlveda nous paraît évident, celui de Las Casas, bien que dissemblable, existe tout autant. Ce dernier ne rejette pas les coutumes amérindiennes, mais les défend à l'aune de ce qui est justifiable dans sa propre culture. II n'est conséquemment ni dans la négation totale de l'Autre par assimilation à soi, ni dans le rejet total de l'Autre pour ses différences. Cependant, les critères à l'origine de sa défense sont tous issus de l'analogie entre l'Autre et soi. Imaginons le cas où cette analogie n'est plus possible (ou jugée inenvisageable) : il serait contraint de percevoir et condamner l'étranger à l'aune de sa forte dissemblance. C'est d'ailleurs exactement ce qui s'est produit lorsque Las Casas a proposé à plusieurs reprises (dans des écrits en 1518 et 1522, dans une lettre au Conseil des Indes en 1531 puis à nouveau en 1542 dans un plan général de réformes) comme remède à l'esclavage amérindien son remplacement par celui des Noirs africains qui, eux, étaient jugés plus robustes, sauvages et pouvant correspondre à l'infériorité naturelle conceptualisée par Aristote (Saint-Lu, 1992). Si la légende noire selon laquelle Las Casas serait l'un des responsables de la traite négrière n'est pas véridique historiquement, et bien qu'il ait lui-même amèrement regretté ses demandes en ce sens, ces textes témoignent néanmoins de cette possibilité : lorsque l'analogie de l'Autre à soi est jugée impossible, l'Autre peut potentiellement être démuni de toute dignité. Ses écrits tardifs critiques à l'égard de la traite négrière témoignent cependant que le cas personnel de Las Casas est à nuancer.

Deuxième point: l'assimilationisme sous-tendu par l'argumentation du prêtre dominicain. L'Autre étant essentiellement semblable à soi, il est dès lors possible et même nécessaire d'un point de vue théologique de le mener - par des voies pacifiques - à l'assimilation par la conversion. Autrement dit, la défense, y compris de l'enjeu polémique du cannibalisme, n'est pas faite au nom du respect de l'étranger dans son étrangeté, mais de l'étrangeté comme étant potentiellement aplanie, et l'étranger rendu ainsi assimilable à soi.

Enfin, la vision arasante, par son souci légitime de mise à égalité, est soumise constamment au risque de s'aveugler sur certains points épineux en les omettant totalement du discours ou au risque de les justifier, comme le fait en un certain sens Las Casas avec la question des sacrifices humains ou du cannibalisme. Rappelons par exemple que si certains sacrifices pouvaient s'appliquer à des enfants préalablement drogués et frappés à mort, étranglés ou encore enterrés vivants, la pratique cannibale est quant à elle plus ambivalente, quoique elle ait pu, elle aussi, résulter d'un meurtre, notamment de l'ennemi. Ainsi, lorsque l'analogie tissant des liens entre l'Autre et soi n'est plus possible, lorsque les différences sont trop prononcées, soit on les omet, soit on tente de les expliquer. La rationalisation nécessaire à la compréhension peut parfois basculer vers la justification, la frontière entre

\footnotetext{
${ }^{15}$ Cependant il écrit aussi : "Mais qui pourrait nier ou douter, à moins d'être idolâtre, qu'anéantir l'idolâtrie serait un juste objectif si elle pouvait être détruite sans scandale, sans danger, sans dommage et sans inconvénient pour les sujets et les non sujets ? " (Capdevila, 2007, p. 257).
}

Alterstice - Revue Internationale de la Recherche Interculturelle, vol. 5, $n^{\circ} 1$ 
justification et explication étant extrêmement poreuse: lorsque par exemple le banquet anthropophage est qualifié de " réjouissant ${ }^{16}$, cette frontière semble allègrement franchie.

S'il nous semble important de souligner ces faiblesses dans la vision de Las Casas, ce n'est évidemment pas pour juger ce personnage historique crucial, mais pour mettre en lumière les limites de ce que l'on a intitulé la vision arasante.

\section{Conclusion : l'anthropophagie comme archétype}

Que penser et que faire face aux comportements radicalement différents des étrangers, surtout lorsque ceux-ci sont condamnés moralement? C'est aussi à cette question que répondent les deux protagonistes de Valladolid. L'étude de la controverse nous a permis de présenter deux visions opposées concernant l'altérité amérindienne, les prémisses sur lesquelles se fondait chacun des protagonistes expliquant leurs regards diamétralement distincts sur le cannibalisme. Ces regards forment certainement le point culminant de la controverse : I'anthropophagie est comme un archétype à partir duquel tout se joue. La réflexion entourant cette controverse nous a permis - outre une compréhension intrinsèque d'un événement historique capital - de développer un modèle dialectique où deux visions de l'altérité s'opposent, chacune étant susceptible de devenir source de richesse et d'affliction. Les rencontres interculturelles sont constamment soumises à cette question: comment réagir face à l'étrangeté, jusqu'où l'Autre doit-il être assimilé à soi et dans quelle mesure cette assimilation peut-elle aussi être une menace? Autrement dit est posée ici la question du rejet ou non de la différence, et bien que dans cette controverse la position de Las Casas ait vraisemblablement eu des répercussions positives sur le cours de l'Histoire, son argumentaire comprend certaines zones d'ombre.

Dans "Paix et proximité ", Levinas décrit avec une grande lucidité l'ampleur de la révolution intellectuelle qui est exigée pour penser la différence dans l'égalité :

On doit précisément remettre en question la conception selon laquelle, dans la multiplicité humaine, l'Ego peut être réduit à une partie du Tout [...] dont l'unité est la cohésion de ses membres ou de sa structure globale. Il est nécessaire de se demander si l'altérité d'autrui n'a pas un caractère absolu [...] si la paix, alors, au lieu d'être le résultat d'une absorption ou d'une disparition de l'altérité, ne serait pas au contraire le mode fraternel d'une proximité à autrui ». (Levinas, 1984, p. 144)

L'équilibre serait donc, dans le regard porté sur l'altérité, un " mode fraternel d'une proximité à autrui », où les particularités d'autrui n'abolissent pas la proximité ni cette dernière ne cache la véritable et nécessaire altérité.

\section{Références}

Auger, D., Rosellini, M., SaÏd, S. et Vidal-Naquet, P. (1979). Aristophane, Les femmes et la cité. Paris : ENS Éditions. (Cahiers de Fontenay 17).

Bénat-Tachot, L., Lavallé, B. (2005). L'Amérique de Charles Quint. Bordeaux : Presses universitaires de Bordeaux.

Capdevila, N. (2007). La controverse entre Las Casas et Sepulveda, précédé de Impérialisme, empire et destruction. Paris : Vrin.

Détienne, M. (1996). Dionysos mis à mort. Paris : Gallimard.

Dickinson J. et Mahn-Lot, M. (1991). Les Européens découvrent l'Amérique : 1492-1992. Lyon : Presses universitaires de Lyon.

Dorel, F. (2006). La thèse du "génocide indien » : guerre de position entre science et mémoire. Amnis. Revue de civilisation contemporaine Europes/Amériques, 6.

Graulich, M. (2005). Le Sacrifice humain chez les Aztèques. Paris : Fayard.

\footnotetext{
${ }^{16}$ Les cérémonies sacrificielles, pour leur part, « quoique cruelles, étaient minutieuses, subtiles et raffinées » (Capdevila, 2007, p. 190 et p. 193).
} 
Guilles-Escuret, G. (2012). Les mangeurs d'autres, civilisation et cannibalisme. Paris : Éditions de l'École des hautes études en sciences sociales.

Las Casas, B. (1979). Très brève relation de la destruction des Indes. Paris : Maspero.

Las Casas, B. (1991). Barthélémy de Las Casas, L'Évangile et la Force. Présentation, choix de textes et traduction par Marianne Mahn-Lot. Paris : Éditions du Cerf.

Las Casas, B. (2002). Histoire des Indes (volume 3, traduit par J.-P. Clément et J.-M. Saint-Lu). Paris : Seuil.

Lavallé, B. (2007). Bartholomé de Las Casas : entre l'épée et la croix. Paris : Payot.

Levinas, E. (1984). Paix et proximité. Dans Cahiers de la nuit surveillée. Paris : Verdier.

Lévinas, E. (2000). Totalité et Infini. Paris : Biblio-Essais.

Hanke, L. (1957). Colonisation et conscience chrétienne. Paris : Plon.

Mahn-Lot, M. (1991). Bartolomé de Las Casas, une théologie pour le Nouveau Monde. Paris : Desclée de Brouwer.

Russel T. (1990). American Indian Holocaust and Survival. A Population History since 1492. Oklahoma : University of Oklahoma Press.

Saint-Lu, A. (1992). Bartolomé de las Casas et la traite des nègres. Bulletin hispanique, 94(1), 37-43.

Schnapp, A. (1997). Le Chasseur et la cité : chasse et érotique en Grèce ancienne. Paris : Albin Michel.

Todorov, T. (1982). La conquête de l'Amérique : la question de l'autre. Paris : Seuil. 